\title{
Laparoendoscopic Single-Site Combined Hysterectomy and Nephrectomy in Deep Infiltrating Endometriosis
}

\author{
Merve Biçer1', Murat Arslan², Vedat Ulug1, Zekeriya Güner'1, Suleyman Akarsu1, Mert Gol ${ }^{3}$ \\ ${ }^{1}$ Department of Obstetrics and Gynecology, Izmir University Hospital, Izmir, Turkey \\ ${ }^{2}$ Department of Urology, Izmir University Hospital, Izmir, Turkey \\ ${ }^{3}$ Private Gynecology Clinic, Izmir, Turkey \\ Email: *mervebicermd@gmail.com
}

Received 22 September 2015; accepted 22 December 2015; published 25 December 2015

Copyright (C) 2015 by authors and Scientific Research Publishing Inc.

This work is licensed under the Creative Commons Attribution International License (CC BY). http://creativecommons.org/licenses/by/4.0/

(c) (;) Open Access

\begin{abstract}
Within the advance ports and instruments, laparoendoscopic single-site (LESS) surgery has been increasingly used among gynaecologic surgeons. In addition, advanced port systems have enabled to perform concomitant surgical procedures via transumbilical incision. However, combined surgical operations are rarely reported in the literature, particularly in gynaecology. This case report presents a 42-year-old woman who undergo concomitant LESS hysterectomy and nephrectomy due to deep infiltrating endometriosis (DIE) and end stage renal disease. The operation is performed in approximately 3 hours without any complications, and the patient is discharged on postoperative third day. This case demonstrates that complex concomitant operations even in a patient with DIE can be performed using a LESS surgical approach.
\end{abstract}

\section{Keywords}

\section{Laparoendoscopic Single-Site (LESS) Surgery, Endometriosis}

\section{Introduction}

Laparoendoscopic single-site (LESS) surgery has been developed as a less-invasive alternative to traditional laparoscopic surgery using several ports. LESS surgery offers the benefits of improved cosmesis, and potential benefits of decreased pain and improved recovery. Accordingly, it has become increasingly widespread and has gained much attention in the recent years among gynaecologic surgeons [1] [2].

\footnotetext{
${ }^{*}$ Corresponding author.
}

How to cite this paper: Biçer, M., Arslan, M., Ulug, V., Güner, Z., Akarsu, S. and Gol, M. (2015) Laparoendoscopic Single-Site Combined Hysterectomy and Nephrectomy in Deep Infiltrating Endometriosis. International Journal of Clinical Medicine, 6, 940-943. http://dx.doi.org/10.4236/ijcm.2015.612123 
When a suitable case of multiple pathologies is encountered, combined laparoscopic procedures may be needed. Combining surgeries may save patients from multiple operations, exposure to recurrent anaesthesia and preoperative examinations. Accordingly, use of universal single laparoscopic port among multiple surgical specialities placed in the umbilicus may offer the advantage of combining multiple operations through a single incision. However, there is limited information about the application of combined laparoscopic operations using LESS surgery.

With the development of minimally invasive surgery, we report the first case in the literature that has undergone concomitant laparoscopic hysterectomy and nephrectomy procedure performed using the LESS surgical approach.

\section{Case}

A 42-year-old woman with a gynecologic history of gravida 2, para 2, has been suffering from chronic pelvic pain, dysmenorrhea, and dyspareunia during past 10-years. She informed that the pain was extanding from the left inguinal region through the left costo-vertebral region. However, she did not have any diagnosis regarding chronic pelvic pain and dyspareunia for years. She reported a history of two normal vaginal deliveries. She denied any other important medical or surgical history.

Pelvic examination demonstrated a 10 -week-sized uterus that was retroverted and semi-mobile. At pelvic ultrasound, uterus was measured $8 \times 4 \times 4.6 \mathrm{~cm}$ with a $4 \times 3.2 \mathrm{~cm}$ intramural fibroid. The right adnexa seemed normal whereas there was an endometrioma in the left ovary measuring $3.2 \times 4 \mathrm{~cm}$. Both ovaries were located behind the uterus. Preoperative laboratory examinations were in normal range except $70 \mathrm{mUI} / \mathrm{L}$ of Ca-125. The intravenous pyelogram showed a normally functioning right kidney and non-functioning left kidney. Tc-DTPA examination revealed no function in the left kidney and normal function in the right kidney. MR examination showed an atrophic left kidney and obliterated rectovaginal septum by the rectum and ovaries. There was no visible endometriotic nodule detected by transvaginal sonographic examination or MR. After evaluation she had preoperative diagnosis of non-functioning atrophic left kidney which is so called end stage kidney due to the presumed diagnosis of deep infiltrating endometriosis. After extensively counseling of the patient about the various laparoscopic techniques available to perform a she opted to undergo a combined surgical procedure, consisting of a concomitant laparoscopic nephrectomy and total laparoscopic hysterectomy via a single umbilical incision. The patient positioned 45 degrees left lateral decubit position and was fixed to the table with plaster. After nephrectomy her position was changed to standart supine position. The nephrectomy procedure we performed's fulfill umblicus-LESS while the same SILS port (covidien) for nephrectomy and hysterectomy is used.

Hysterectomy was performed with the assistance of a uterine manipulator containing a pneumo-occluder balloon and colpotomy ring (RUMI II Uterine Manipulator and KOH Colpotomizer; Cooper Surgical, Inc., Izmir, TR.) and using a single-incision laparoscopic surgical port (Covidien, Izmir, Tr). Figure 1 shows the laparoscopic view at the beginning of the surgery.

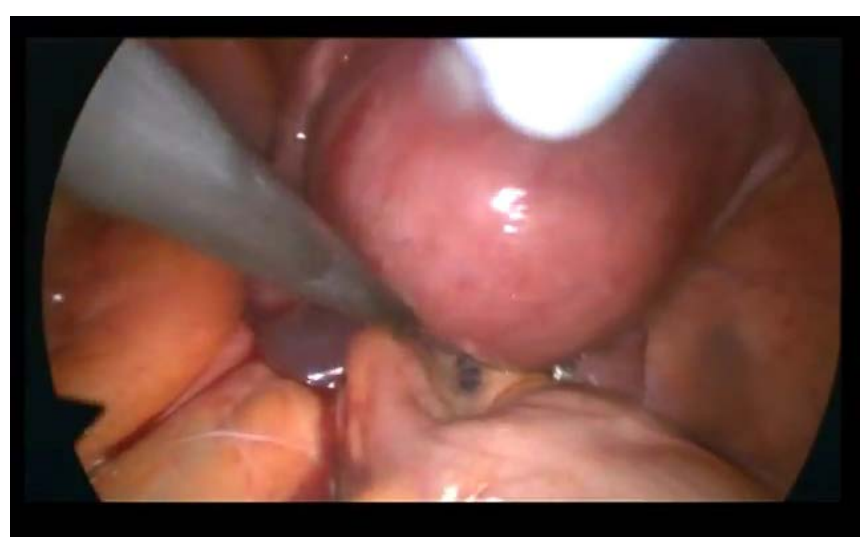

Figure 1. Optical view during the beginning of the procedure. Note that the sigma and the rectum are completely adherent behind the uterus and ovaries can not be seen. 


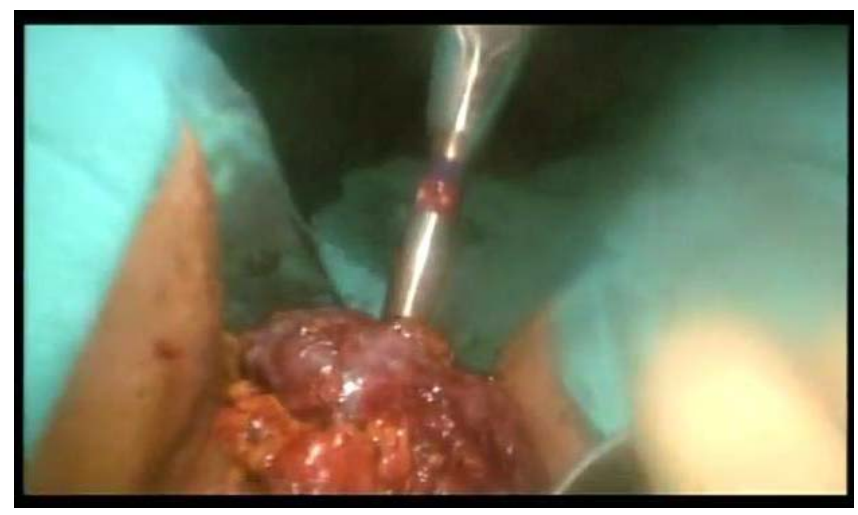

Figure 2. Removal of the left kidney through the vagina.

The sigmoid colon and the rectum were completely adherent behind the uterus where ovaries were not easily seen. The sigmoid colon was detached from the uterus using a sharp dissection. Accordingly, both ovaries were dissected and separeted from the posterior wall of the uterus. Rectovaginal fould was also opened using sharp dissection. No significant retrocervical nodüle was seen. However there was an endometriotic tissue on the anterior surface of the rectum which was pathologically confirmed. Proceedingly laparoscopic hysterectomy was performed as published before [3]. Then through the same single port left nephrectomy was performed and the kidney was removed from the vagina (Figure 2). The vagina was sutured vaginally. The abdomen and pelvis were thoroughly irrigated, and hemostasis was confirmed. The single-incision laparoscopic surgery port was removed, and the fascia was closed with a delayed absorbable suture in running fashion. Total operation time was 160 minutes and total intraoperative bleeding was 150 cc. The patient was discharged on the third postoperative day without any complaint. Pathologic analysis demonstrated a 140-g uterus with multiple intramural leiomyomas, and an atrophic left kidney.

\section{Discussion}

LESS surgery presents challenges for the surgeon because of loss of instrument triangulation, reduced operating space, and increased exhaustion of the surgeon during long procedures. Therefore, laparoscopic surgeons should have trained extensively for this difficult and different technique. Moreover, combined laparoscopic surgical operations using transumbilical incision are seldom done and need extensive knowledge of the laparoscopic anatomy.

The first published combined technique using LESS showed the feasibility of performing hysterectomy and cholecystectomy [4]. Subsequently, a recent case series by Palanivelu et al. [5] reported a retrospective analysis of 27 patients in whom various combined laparoscopic single site operations had been performed. In their series, there were 2 patients in whom hysterectomy operation was combined with sleeve gastrectomy, and cholecystectomy, respectively via single route. They reported a time of 140 minutes for each operation without any complications.

Deep infiltrating endometriosis (DIE) surgery is a challenging procedure even in skilful hands. In most of these patients, pouch of Douglas is obliterated with rectum and ovaries. In addition, there are dense adhesions between the uterus and the rectum which makes it difficult to open the rectovaginal septum thus enhancing the risk of rectal damage. In the present case, the pouch of Douglas is completely obliterated with the rectum and detachment is obtained by using sharp dissection. No evident retro cervical nodule is seen but endometriotic tissue that is confirmed by pathology is removed from the anterior surface of the rectum (Shaving technique). In some of the patients with DIE, the incidence of ureteric involvement and silent renal loss may be as high as up to $1.5 \%$ [6]. Accordingly, in patients with DIE over the age of 40, removal of the uterus and the ovaries may be a curable option. Therefore, combined operations as hysterectomy, ureterolysis, end to end ureter anastomosis, and nephrectomy may be needed in these patients.

A previous article by Kijvikai et al. [7] reported a patient with end stage renal disease due to DIE. The patient undergone combined laparoscopic nephrectomy and hysterectomy. In these patient authors used three 10-mm ports for nephrectomy and additionally three $5-\mathrm{mm}$ and one 10-mm ports for laparoscopic hysterectomy with a 
total of 7 ports. We considered that combining laparoscopic hysterectomy and nephrectomy by only using a 3 -cm umbilical incision offered the advantage of improved cosmetic result, less pain, and improved recovery in the present patient.

To our knowledge, the present case is the first in the literature that LESS nephrectomy and hysterectomy are performed in a patient with DIE and end-stage renal disease. We suggest that combined complex concomitant surgical procedures can be performed safely and efficiently using LESS surgery if the surgeons improve their surgical skills and techniques. This case demonstrates the feasibility of combining urologic and gynaecologic procedures concomitantly by using single umbilical incision.

\section{Competing Financial Interests}

No competing financial interests exist.

\section{References}

[1] Wenger, J.M., Dubuisson, J.B. and Dällenbach, P. (2012) Laparoendoscopic Single-Site Supracervical Hysterectomy with Endocervical Resection. Journal of Minimally Invasive Gynecology, 19, 217-219. http://dx.doi.org/10.1016/j.jmig.2011.10.009

[2] Behnia-Willison, F., Foroughinia, L., Sina, M. and McChesney, P. (2012) Single Incision Laparoscopic Surgery (SILS) in Gynaecology: Feasibility and Operative Outcomes. Australian \& New Zealand Journal of Obstetrics \& Gynaecology, 52, 366-370. http://dx.doi.org/10.1111/j.1479-828X.2012.01443.x

[3] Gol, M., Kizilyar, A. and Eminoglu, M. (2007) Laparoscopic Hysterectomy with Retroperitoneal Uterine Artery Sealing Using ligasure: Gazi Hospital Experience. Archives of Gynecology and Obstetrics, 276, 311-314. http://dx.doi.org/10.1007/s00404-007-0353-1

[4] Hart, S., Ross, S. and Rosemurgy, A. (2010) Laparoendoscopic Single-Site Combined Cholecystectomy and Hysterectomy. Journal of Minimally Invasive Gynecology, 17, 798-801. http://dx.doi.org/10.1016/j.jmig.2010.07.006

[5] Palanivelu, C., Ahluwalia, J.S., Palanivelu, P., Palanisamy, S. and Vij, A. (2013) Combined Surgical Procedures Using Laparoendoscopic Single-Site Surgery Approach. Asian Journal of Endoscopic Surgery, 6, 165-169. http://dx.doi.org/10.1111/ases.12023

[6] Miranda-Mendoza, I., Kovoor, E., Nassif, J., Ferreira, H. and, Wattiez, A. (2012) Laparoscopic Surgery for Severe Ureteric Endometriosis. European Journal of Obstetrics \& Gynecology and Reproductive Biology, 165, 275-279. http://dx.doi.org/10.1016/j.ejogrb.2012.07.002

[7] Kijvikai, K., Srisombut, C. and Tingthanatikul, Y. (2007) Laparoscopic Nephrectomy, Hysterectomy and Rectovaginal Endometriotic Mass Removal in a Single Session. Journal of the Medical Association of Thailand, 90, 1934-1936. 